\section{Neoadjuvant targeted therapy in renal cell carcinoma}

\author{
Roberto lacovelli, Cristina Raimondi, Antonella Palazzo, Enrico Cortesi \\ and Giuseppe Procopio
}

We read with interest the article published by Vincenzo Ficarra and Giacomo Novara Kidney cancer: neoadjuvant targeted therapies in renal cell carcinoma. Nat. Rev. Urol. 7, 63-64; 2010). ${ }^{1}$ In the past few years, randomized trials have shown the efficacy of targeted therapies in renal cell carcinoma (RCC); these results have dramatically changed the therapeutic approach. It is important to draw attention to the following issues.

First, the appropriateness of using TNM staging to determine eligibility of patients for neoadjuvant treatment. It has been shown that the TNM system is not sufficient for risk stratification of patients with resected or advanced RCC. A combination of clinical and histological factors, such as TNM staging, Fuhrman's tumor grade and Eastern Cooperative Oncology Group (ECOG) performance status, have more relevant prognostic value in terms of survival, according to the UCLA Integrated Staging System (UISS). ${ }^{2}$

Patients with nonmetastatic highrisk disease have a high rate of systemic failure, suggesting the presence of occult metastases at diagnosis that are not detectable using current imaging methods. Moreover, similar survival curves have been published for nonmetastatic high-risk patients and metastatic low-risk patients. ${ }^{3}$

Neoadjuvant therapy is commonly used in an effort to downstage locally advanced tumors, as well as to improve survival. Parameters in current use-including large tumor size, involvement of vital structures, and bulky lymphadenopathy-may not be sufficient to identify patients who are eligible for medical treatment. Precisely defining the UISS risk class could be more useful in the presurgical decisionmaking phase. Considering the lack of data supporting adjuvant treatments in patients with RCC, neoadjuvant medical therapy that has the effect of delaying nephrectomy could facilitate earlier onset of systemic disease control.

Considerable doubt remains regarding the poor response rate of primary tumors treated with targeted agents, relative to the higher overall response rate reported in a number of phase III trials of bevacizumab plus interferon, or sunitinib, in metastatic RCC.

$91 \%$ of patients treated with sunitinib in phase III trials had previously undergone nephrectomy; as such, comparable data on primary tumor shrinkage are not available. ${ }^{4}$

It is possible that the poor response of primary tumors, as published in preliminary reports, is not a function of limited efficacy of anti-angiogenic treatment. Rather, it might be ascribed to a reduced ratio of neoplastic tissue to tumor necrosis, which is usually present at the center of the primary disease site. ${ }^{5}$ The superior response rate observed in the metastatic setting could be a function of the higher burden of non-necrotic tumor.

In the neoadjuvant setting, a $15 \%$ reduction in tumor size is not considered an exciting result, even though a mean size decrease of $10 \%$ has been reported to be an optimal criterion for identification of patients with metastatic RCC who will derive benefit from sunitinib treatment in terms of progression-free and overall survival. ${ }^{6,7}$

Whether the achieved degree of tumor downsizing is sufficient to facilitate surgery is an unresolved issue, as is the optimal timing of neoadjuvant treatment.

In conclusion, the rapidity of recent advances in RCC management make it difficult to establish a therapeutic algorithm that accounts for the numerous and evolving prognostic/predictive factors. The use of targeted therapies in a neoadjuvant setting, with the aims of significantly reducing tumor volume before surgery and gaining better control of disease in high-risk patients, is a fascinating future option. These theoretical advantages could balance the published possible risk of perioperative complications associated with neoadjuvant anti-angiogenic therapies.

Department of Experimental Medicine, Oncology Unit, "Sapienza" University of Rome, Viale Regina Elena 324, 00161 Rome, Italy (R. lacovelli, C. Raimondi, A. Palazzo, E. Cortesi). Medical Oncology 2 Unit, Fondazione Istituto Nazionale dei Tumori, Via Giacomo Venezian 1, 20133 Milan, Italy (G. Procopio).

Correspondence to: $R$. lacovelli roberto.iacovelli@alice.it

doi:10.1038/nrurol.2010.2-c1

Competing interests

The authors declare no competing interests.

1. Ficarra, V. \& Novara, G. Kidney cancer: neoadjuvant targeted therapies in renal cell carcinoma. Nat. Rev. Urol. 7, 63-64 (2010).

2. Zisman, A. et al. Risk group assessment and clinical outcome algorithm to predict the natural history of patients with surgically resected renal cell carcinoma. J. Clin. Oncol. 20, 4559-4566 (2002).

3. Belldegrun, A. S. et al. Cancer-specific survival outcomes among patients treated during the cytokine era of kidney cancer (1989-2005): a benchmark for emerging targeted cancer therapies. Cancer 113, 2457-2463 (2008).

4. Motzer, R. J. et al. Sunitinib versus interferon alfa in metastatic renal-cell carcinoma. N. Engl. J. Med. 356, 115-124 (2007).

5. van der Veldt, A. A. et al. Sunitinib for treatment of advanced renal cell cancer: primary tumor response. Clin. Cancer Res. 14, 2431-2436 (2008).

6. van der Veldt, A. A., Meijerink, M. R., van den Eertwegh, A. J., Haanen, J. B. \& Boven, E. Choi response criteria for early prediction of clinical outcome in patients with metastatic renal cell cancer treated with sunitinib. Br. J. Cancer 102, 803-809 (2010).

7. Thiam, R. et al. Optimizing the size variation threshold for the CT evaluation of response in metastatic renal cell carcinoma treated with sunitinib. Ann. Oncol. 21, 936-941 (2010). 